\title{
A STOCHASTIC INVENTORY MODEL WITH PERISHABLE AND AGING ITEMS
}

\author{
LAKHDAR AGGOUN and LAKDERE BENKHEROUF \\ Sultan Qaboos University \\ Department of Mathematics and Statistics, College of Science \\ P.O. Box 36 Alkhod \\ Muscat 123, Oman \\ LOTFI TADJ \\ King Saud University \\ Department of Statistics and Operations Research \\ College of Science, P.O. Box 2455 \\ Riyadh 11451, Saudi Arabia
}

(Received May, 1997; Revised May, 1998)

\begin{abstract}
In this paper, we propose a single-product, discrete time inventory model for perishable items. Inventory levels are reviewed periodically and units in stock have a maximum lifetime of $M$ periods. It is assumed that the dynamics of the inventory level is driven by a parameter process (reflecting perishability) and demands. By observing the history of the inventory level we obtain the conditional distribution of the perishability parameter by using the change of measure techniques. A special case is also presented.
\end{abstract}

Key words: Inventory Control, Perishable Items, Markov Models, Change of Measure Techniques.

AMS subject classifications: $60 \mathrm{~K} 30,60 \mathrm{~J} 10,90 \mathrm{~B} 05$.

\section{Introduction}

In this paper we propose a single-product, discrete time inventory model for perishable items. Inventory levels are reviewed periodically and units in stock have a maximum useful life of $M$ periods. The state vector $X_{n}=\left(X_{n}^{0}, X_{n}^{1}, \ldots, X_{n}^{M}\right)$ gives the inventory level of each category, where $X_{n}^{i}$ is a $\mathbb{Z}^{+}$-valued process representing the inventory level in period $n$ of items of age $i, i=0,1, \ldots, M$. Items older than $M$ periods are discarded. Each item of age $i<M$ is assumed to survive to the next period with probability $\theta_{n}^{i}$ or perish with probability $\left(1-\theta_{n}^{i}\right), i=0,1, \ldots, M$ and $n=$ $0,1, \ldots$. The subscript $n$ in $\left(\theta_{n}^{i}\right)$ reflects a dependence of the perishability coefficient on period $n$. It is well known, for example, that weather conditions have some effect on perishability of vegetables and hence the importance of the dependence of $\left(\theta_{n}^{i}\right)$ on 
n. Further, we assume that the demand at time $n$ is represented by $M+1, \mathbb{Z}^{+}$valued, random variables $V_{n}^{0}, V_{n}^{1}, \ldots, V_{n}^{M}$ with probability distributions $\phi_{0}, \phi_{1}, \ldots, \phi_{M}$, respectively. We also suppose that newly arrived items have age zero and that replenishment is allowed in each period. Let $U_{n}$ be the $\mathbb{Z}^{+}$-valued replenishment process, which is assumed to be measurable with respect to the history up to to time $(n-1)$ of the inventory level. Mathematically, the inventory level follows the dynamics:

$$
\begin{gathered}
X_{n}^{0}=U_{n}-V_{n}^{0} \\
X_{n}^{i}=\left(\theta_{n-1}^{i-1}\right) \circ X_{n-1}^{i-1}-V_{n}^{i}, \quad i=1, \ldots, M .
\end{gathered}
$$

The operator "o" in (1.2) reflects the notion of perishability alluded to above and is defined for $X_{n-1}^{i-1}>0$ as:

$$
\theta_{n-1}^{i-1} \circ X_{n-1}^{i-1}=\sum_{j=1}^{X_{n-1}^{i-1}} Y_{j}^{n, i}
$$

where $Y_{j}^{n, i}$ is a sequence of random variables independent of $X_{n-1}^{i-1}$, with conditional distribution

$$
P\left(Y_{j}^{n, i}=1 \mid \theta_{n-1}^{i-1}=\theta\right)=\theta, P\left(Y_{j}^{n, i}=0 \mid \theta_{n-1}^{i-1}=\theta\right)=1-\theta .
$$

Note that we allow for $X_{n-1}^{i-1}$ to take negative values, in which case $\theta_{n-1}^{i-1} \circ X_{n-1}^{i-1} \equiv 0$ in (1.3). In this case, $X_{n-1}^{i-1}$ may be interpreted as a shortage.

We assume that $\left\{\theta_{n}^{i}\right\}, i=0,1, \ldots, M$, are Markov processes with the common state space as the interval $(0,1)$, with the initial distributions $\left\{\pi_{0}^{i}\right\}(i=0,1, \ldots, M)$ and transition densities:

$$
P\left[\theta_{n}^{i} \in d z \mid \theta_{n-1}^{i}=\theta\right]=\rho_{n, i}(\theta, z) d z .
$$

We assume that $\theta_{n}^{i}$ is stochastically larger than $\theta_{n}^{i+1}$ for all $i=0,1, \ldots, M-1$. This seems intuitively plausible, since older items are more likely to perish than younger ones.

Models, using the operator "o" as defined in (1.3), have been used for analysis of special types of time series by Al-Osh and Alzaid [1] and McKenzie [4]. The interest there was on modeling of counting processes of dependent variables.

By observing the history of the inventory levels, we are interested in deriving the conditional joint distributions of the Markov processes $\left\{\theta_{n}^{i}\right\}$ the perishability phenomenon.

Examples where items experience some kind of perishability or deterioration include food products, blood samples, drugs, electronic components, etc. Models for deteriorating items have existed for some time now. For deterministic models, the review of Raafat [8] gives a good account of existing work. For stochastic models, Nahmias [6] may be consulted. For the stochastic models with fixed lifetimes, good progress has been made in obtaining an optimal replenishment schedule for some models, see Nandakumar and Morton [7]. However, when the lifetime of the items is random, difficulties arise in the analysis and there seems to be only instances of the study of $(S-1, S)$ policies using queueing models, see Moinzadeh [5] and Smeitink [9] and the references therein. 
In Section 2, using the measure change technique (see Elliot, Aggoun, and Moore [3]), recursive unnormalized densities are derived. Section 3 contains a special case where the transition densities of the Markov processes are assumed to be conditional normal densities. We conclude the paper with some general remarks.

\section{Densities Estimation}

In this section, a reference probability measure, under which all the random variables of interest are independent, is constructed.

All random variables are initially defined on a probability space $\{\Omega, \mathcal{F}, P\}$. Recall from Section 1 that $X_{n}^{i}$ represents the inventory level at time $n$ of age $i$ and $\theta_{n}^{i}$ represents the perishability parameter. Now, let

$$
\begin{gathered}
\Theta_{n}=\sigma\left\{\theta_{k}^{i} ; i=0,1, \ldots, M, k \leq n\right\}, \\
\mathscr{F}_{n}=\sigma\left\{X_{k}^{i}, Y_{j}^{k, i} ; i=0,1, \ldots, M, j \geq 1, k \leq n\right\},
\end{gathered}
$$

and

$$
G_{n}=\sigma\left\{X_{k}^{i}, \theta_{k}^{i}, Y_{j}^{k, i} ; i=0,1, \ldots, M, j \geq 1, k \leq n\right\}
$$

be the complete filtrations generated by the parameter process $\left\{\theta_{k}^{i}\right\}$, the inventory level-surviving items process $\left\{X_{k}^{i}, Y_{j}^{k, i}\right\}$, and the inventory level-surviving items process and the parameter process, respectively. Note that $\Theta_{n}$ and $\mathcal{F}_{n}$ are included in $G_{n}$ for $n=1, \ldots$.

For some suitable positive densities $\psi_{i}$ define, for $k \geq 1$,

$$
\lambda_{k}=\prod_{i=0}^{M} \frac{\phi_{i}\left(X_{k}^{i}\right)}{\phi_{i}\left(V_{k}^{i}\right)} \frac{\psi_{i}\left(\theta_{k}^{i}\right)}{\rho_{k, i}\left(\theta_{k}^{i}, \theta_{k-1}^{i}\right)}
$$

where $\phi_{0}, \phi_{1}, \ldots, \phi_{M}$ are the probability distributions of $V_{n}^{0}, V_{n}^{1}, \ldots, V_{n}^{M}$, respectively, and $\rho_{k, i}$ 's are defined in (1.5). Note that

$$
E\left[\lambda_{n} \mid G_{n-1}\right]=1
$$

where $G_{n}$ is given by (2.3) and $E$ denotes the expectation under $P$. Define

$$
\Lambda_{n}=\prod_{k=0}^{n} \lambda_{k}
$$

Then $\left\{\Lambda_{n}\right\}$ is a $G_{n}$-martindale such that $E\left[\Lambda_{n}\right]=1$. We can define a new probability measure $P^{*}$ on $\left(\Omega, \underset{n}{\stackrel{\infty}{V}} G_{n}\right)$ by setting

$$
\left.\frac{d P^{*}}{d P}\right|_{G_{n}}=\Lambda_{n}
$$

The existence of $P^{*}$ on $\left(\Omega \stackrel{\infty}{\stackrel{\infty}{v}} G_{n}\right)$ follows from Kolmogorov's extension theorem (see e.g., Chung [2]). The next lemma shows that under $P^{*},\left\{\theta_{k}^{i}\right\}$ is a sequence of in- 
dependent random variables with probability densities $\psi_{i}$. Similarly, $\left\{X_{k}^{i}\right\}$ forms a sequence of independent random variables with probability distribution $\phi_{i}(\cdot), i=0,1$, $\ldots, M$.

Lemma 1: Under the new probability measure $P^{*}$ defined in (2.5) $\left\{\theta_{k}^{i}\right\}$ are independent with densities $\psi_{i}$ and $\left\{X_{k}^{i}\right\}$ are independent with the common distribution $\phi_{i}(\cdot), i=0,1, \ldots, M$.

Proof: Let $\left\{f_{i}\right\}$ and $\left\{h_{i}\right\}, i=0, \ldots, M$ be integrable test functions. Consider

$$
E^{*}\left[\prod_{i=0}^{M} f_{i}\left(\theta_{k}^{i}\right) h_{i}\left(X_{k}^{i}\right) \mid G_{n-1}\right]=\frac{E\left[\prod_{i=0}^{M} f_{i}\left(\theta_{k}^{i}\right) h_{i}\left(X_{k}^{i}\right) \Lambda_{n} \mid G_{n-1}\right]}{E\left[\Lambda_{n} \mid G_{n-1}\right]},
$$

where $E^{*}$ denotes the expectation under $P^{*}$. Now using the fact that $\Lambda_{n}$ is a $G_{n^{-}}$ martingale and that $E\left[\Lambda_{n}\right]=1$, due to (2.4), the above is equal to

$$
\begin{gathered}
E\left[\prod_{i=1}^{M} f_{i}\left(\theta_{n}^{i}\right) h_{i}\left(X_{n}^{i}\right) \frac{\phi_{i}\left(X_{n}^{i}\right)}{\phi_{i}\left(V_{n}^{i}\right)} \frac{\psi_{i}\left(\theta_{n}^{i}\right)}{\rho_{n, i}\left(\theta_{n}^{i}, \theta_{n-1}^{i}\right)} E\left[\frac{\psi_{0}\left(\theta_{n}^{0}\right) f_{0}\left(\theta_{n}^{0}\right)}{\rho_{n, 0}\left(\theta_{n}^{0}, \theta_{n-1}^{0}\right)}\left|G_{n}-\left\{\theta_{n}^{0}\right\}\right| G_{n-1}\right]\right] \\
=E\left[\prod_{i=1}^{M} f_{i}\left(\theta_{n}^{i}\right) h_{i}\left(X_{n}^{i}\right) \frac{\phi_{i}\left(X_{n}^{i}\right)}{\phi_{i}\left(V_{n}^{i}\right)} \frac{\psi_{i}\left(\theta_{n}^{i}\right)}{\rho_{n, i}\left(\theta_{n}^{i}, \theta_{n-1}^{i}\right)}\right. \\
\left.\qquad \frac{\psi_{0}\left(t_{0}\right) f_{0}\left(t_{0}\right)}{\rho_{n, 0}\left(t_{0}, \theta_{n-1}^{0}\right)} \rho_{n, 0}\left(t_{0}, \theta_{n-1}^{0}\right) d t_{0} \mid G_{n-1}\right] \\
=E_{\psi_{0}}\left[f_{0}\left(\theta_{n}^{0}\right)\right] E\left[\prod_{i=1}^{M} f_{i}\left(\theta_{n}^{i}\right) h_{i}\left(X_{n}^{i}\right) \frac{\phi_{i}\left(X_{n}^{i}\right)}{\phi_{i}\left(V_{n}^{i}\right)} \frac{\psi_{i}\left(\theta_{n}^{i}\right)}{\rho_{n, i}\left(\theta_{n}^{i}, \theta_{n-1}^{i}\right)}\right] .
\end{gathered}
$$

The same process can be repeated to yield

$$
\begin{gathered}
\prod_{i=0}^{M} E_{\psi_{i}}\left[f_{i}\left(\theta_{n}^{i}\right)\right] E\left[\prod_{i=0}^{M} h_{i}\left(X_{n}^{i}\right) \frac{\phi_{i}\left(X_{n}^{i}\right)}{\phi_{i}\left(V_{n}^{i}\right)} E\left[\frac{h_{0}\left(X_{n}^{0}\right) \phi_{0}\left(X_{n}^{0}\right)}{\phi_{0}\left(V_{n}^{0}\right)}\left|G_{n}-\left\{X_{n}^{0}\right\}\right| G_{n-1}\right]\right] \\
=E\left[\prod_{i=1}^{M} h_{i}\left(X_{n}^{i}\right) \frac{\phi_{i}\left(X_{n}^{i}\right)}{\phi_{i}\left(V_{n}^{i}\right)} \int \frac{h_{0}\left(u_{n}-v_{0}\right) \phi_{0}\left(u_{n}-v_{0}\right)}{\phi_{0}\left(v_{0}\right)} \phi_{0}\left(v_{0}\right) d v_{0} \mid G_{n-1}\right]
\end{gathered}
$$

which leads to

$$
\prod_{i=0}^{M} E_{\psi_{i}}\left[f_{i}\left(\theta_{n}^{i}\right)\right] E_{\phi_{i}}\left[h_{i}\left(X_{n}^{i}\right)\right]
$$

This completes the proof.

Now, write

$$
q_{n}\left(z_{0}, z_{1}, \ldots, z_{M}\right)=E^{*}\left[\prod_{i=0}^{M} I\left(\theta_{n}^{i} \in d z_{i}\right) \Lambda_{n}^{-1} \mid \mathscr{F}_{n}\right]
$$


for the unnormalized conditional joint density of the vector $\left(\theta_{n}^{0}, \ldots, \theta_{n}^{M}\right)$. Note that the normalized conditional density is obtained by simply dividing $q_{n}(\cdot)$ by the normalizing quantity

$$
\int q_{n}\left(z_{0}, z_{1}, \ldots, z_{M}\right) d z_{0} \ldots d z_{M}
$$
Theorem 1: Starting at time 0 with the normalized density $q_{0}=\prod_{i=0}^{M} M_{0}^{i}$, one can
obtain further updates by using the recursion:

$$
\begin{aligned}
q_{n}\left(z_{0}, z_{1}, \ldots, z_{M}\right)= & \frac{\phi_{0}\left(u_{n}-X_{n}^{0}\right)}{\prod_{i=0}^{M} \phi_{i}\left(X_{n}^{i}\right)} \int \prod_{i=0}^{M} \rho_{n, i}\left(z_{i}, w_{i}\right) \prod_{i=0}^{M} \phi_{i}\left(w_{i-1} \circ X_{n-1}^{i-1}-X_{n}^{i}\right) \\
& \times q_{n-1}\left(w_{0}, w_{1}, \ldots, w_{M}\right) d w_{0} \ldots d w_{M} .
\end{aligned}
$$

Proof: Let $f_{0}, \ldots, f_{M}$ be integrable test functions. Using (2.6),

$$
E^{*}\left[\prod_{i=0}^{M} f_{i}\left(\theta_{n}^{i}\right) \Lambda_{n}^{-1} \mid \mathscr{F}_{n}\right]=\int \prod_{i=0}^{M} f_{i}\left(z_{i}\right) q_{n}\left(z_{0}, \ldots, z_{M}\right) d z_{0} \ldots d z_{M} .
$$

However,

$$
\begin{gathered}
E^{*}\left[\prod_{i=0}^{M} f_{i}\left(\theta_{n}^{i}\right) \Lambda_{n}^{-1} \mid \mathcal{F}_{n}\right] \\
=E^{*}\left[\Lambda_{n}^{-1} \prod_{i=0}^{M} f_{i}\left(\theta_{n}^{i}\right) \prod_{i=1}^{M} \frac{\phi_{i}\left(\theta_{n-1}^{i-1} \circ X_{n-1}^{i-1}-X_{n}^{i}\right)}{\phi_{i}\left(X_{n}^{i}\right)} \frac{\phi_{0}\left(u_{n}-X_{n}^{0}\right)}{\phi_{0}\left(X_{n}^{0}\right)} \times \frac{\rho_{n, i}\left(\theta_{n}^{i}, \theta_{n-1}^{i}\right)}{\psi_{i}\left(\theta_{n}^{0}\right)} \mid \mathcal{F}_{n}\right] \\
=E^{*}\left[\prod_{i=0}^{M} \frac{\int f_{i}\left(z_{i}\right) \rho_{n, i}\left(z_{i}, \theta_{n-1}^{i}\right)}{\psi_{i}\left(z_{i}\right)} \psi_{i}\left(z_{i}\right) d z_{i} \prod_{i=1}^{M} \frac{\phi_{i}\left(\theta_{n-1}^{i} \circ X_{n-1}^{i-1}-X_{n}^{i}\right)}{\phi_{i}\left(X_{n}^{i}\right)} \Lambda_{n-1}^{-1} \mid \mathcal{F}_{n}\right] \\
=\prod_{i=0}^{M} \frac{1}{\psi_{i}\left(X_{n}^{i}\right)} \iint \prod_{i=0}^{M} f_{i}\left(z_{i}\right) \rho_{n, i}\left(z_{i}, w_{i}\right) \prod_{i=1}^{M} \phi_{i}\left(w_{i-1} \circ X_{n-1}^{i-1}-X_{n}^{i}\right) \\
\times \phi_{0}\left(u_{n}-X_{n}^{0}\right) q_{n-1}\left(w_{0}, \ldots, w_{n}\right) d z_{0} \ldots d z_{M^{d}} d w_{0} \ldots d w_{M} .
\end{gathered}
$$

Comparing (2.7) with (2.8) leads to the required result.

\section{Recursive Parameter Estimation}

Suppose, for simplicity, that the transition densities $\rho_{n, 0}, \ldots, \rho_{n, M}$ are conditional normal densities depending on unknown parameters $\sigma_{0}, \ldots, \sigma_{M}$ :

$$
\rho_{n, i}(\theta, z)=\frac{1}{\sqrt{2 \pi \sigma_{i}}} \exp \left\{-\frac{1}{2}\left(\frac{z-\theta}{\sigma_{i}}\right)^{2}\right\}:=\alpha\left(\theta, z, \sigma_{i}\right) \text {. }
$$


Starting from some prior density functions $h_{0}(\cdot), \ldots, h_{M}(\cdot)$ for $\sigma_{0}, \ldots, \sigma_{M}$, respectively, we wish to update recursively these prior distributions using the information provided by the filtration $\left\{\mathscr{F}_{n}\right\}$. To this end, define:

$$
E^{*}\left[\prod_{i=0}^{M} I\left(\theta_{n}^{i} \in d z_{i}\right) I\left(\sigma_{i} \in d s_{i}\right) \Lambda_{n} \mid \mathscr{F}_{n}\right]:=q_{n}\left(z_{0}, \ldots, z_{M}, s_{0}, \ldots, s_{M}\right)
$$

Now, for arbitrary integrable real-valued test functions $f_{0}, \ldots, f_{M}$ and $g_{0}, \ldots, g_{M}$, we set

$$
\begin{gathered}
E^{*}\left[\prod_{i=0}^{M} f_{i}\left(\theta_{n}^{i}\right) g_{i}\left(\sigma_{i}\right) \Lambda_{n} \mid \mathscr{F}_{n}\right] \\
\quad=\iint \prod_{i=0}^{M} f_{i}\left(z_{i}\right) g_{i}\left(s_{i}\right) q_{n}\left(z_{0}, \ldots, z_{M}, s_{0}, \ldots, s_{M}\right) d z_{0} \ldots d z_{M} d s_{0} \ldots d s_{M} .
\end{gathered}
$$

However,

$$
\begin{gathered}
E^{*}\left[\prod_{i=0}^{M} f_{i}\left(\theta_{n}^{i}\right) g_{i}\left(\sigma_{i}\right) \Lambda_{n} \mid \mathcal{F}_{n}\right] \\
=\iiint \prod_{i=0}^{M} f_{i}\left(z_{i}\right) g_{i}\left(s_{i}\right) \alpha\left(w_{i}, z_{i}, s_{i}\right) \prod_{i=1}^{M} \frac{\phi_{i}\left(w_{i-1} \circ X_{n-1}^{i-1}-X_{n}^{i}\right)}{\phi_{i}\left(X_{n}^{i}\right)} \frac{\phi_{0}\left(u_{n}-X_{n}^{0}\right)}{\phi_{0}\left(X_{n}^{0}\right)} \\
\quad \times q_{n-1}\left(w_{0}, \ldots, w_{M}, s_{0}, \ldots, s_{M}\right) d z_{0} \ldots d z_{M} d s_{0} \ldots d s_{M} d w_{0} \ldots d w_{M}
\end{gathered}
$$

where $\alpha\left(w_{i}, z_{i}, s_{i}\right)$ is given by (3.1). Hence,

$$
\begin{gathered}
q_{n}\left(z_{0}, \ldots, z_{M}, s_{0}, \ldots, s_{M}\right)=\int d w_{0} \ldots d w_{M} \prod_{i=0}^{M} \alpha\left(w_{i}, z_{i}, s_{i}\right) \\
\times \prod_{i=1}^{M} \frac{\phi_{i}\left(w_{i-1} \circ X_{n-1}^{i-1}-X_{n}^{i}\right)}{\phi_{i}\left(X_{n}^{i}\right)} \frac{\phi_{0}\left(u_{n}-X_{n}^{0}\right)}{\phi_{0}\left(X_{n}^{0}\right)} \prod_{i=1}^{M} q_{n-1}\left(w_{0}, \ldots, w_{M}, s_{0}, \ldots, s_{M}\right),
\end{gathered}
$$

and

$$
q_{0}=\prod_{i=0}^{M} \pi_{0}^{i} h_{i}
$$

is the joint distribution, at time 0 , of $\theta_{0}^{i}, \sigma_{i}, i=0, \ldots, M$. Further updates are obtained using (3.3).

\section{Summary}

In this paper, an integer-valued inventory model for perishable items with aging was proposed. It was assumed that the dynamics of the inventory level is driven by a parameter process (reflecting perishability) and demand. By observing the history of 
the inventory level, the conditional distribution of the perishability parameters was derived, by using the change of measure technique.

\section{References}

[1] Al-Osh, M.A. and Alzaid, A.A., First-order integer-valued autoregressive (INAR(1)) processes, J. Time Series Anal. 8 (1987), 261-275.

[2] Chung, K.L., A Course in Probability Theory, Academic Press, New York 1974.

[3] Elliot, R.J., Aggoun, L. and Moore, J.B., Hidden Markov Models: Estimation and Control, Applications of Mathematics 29, Springer-Verlag, New York 1995.

[4] McKenzie, E., Some simple models for discrete variate time series, Water Res. Bull. 21 (1985), 645-650.

[5] Moinzadeh, K., Operating characteristics of the $(S-1, S)$ inventory system with partial backorders and constant supply times, Mgmt. Sci. 35 (1989), 472-477.

[6] Nahmias, S., Perishable inventory theory: A review, Opers. Res. 30 (1982), 680-708.

[7] Nandakumar, P. and Morton, T.E., Near myopic heuristics for fixed life perishability problems, Mgmt. Sci. 39 (1993), 1490-1498.

[8] Raafat, F., Survey of literature on continuously deteriorating inventory models, J. Opl. Res. Soc. 42 (1991), 27-37.

[9] Smeitink, E., A note on operating characteristics of the $(S-1, S)$ inventory system with partial backorders and constant supply times, Mgmt. Sci. 36 (1990), 1413-1414. 


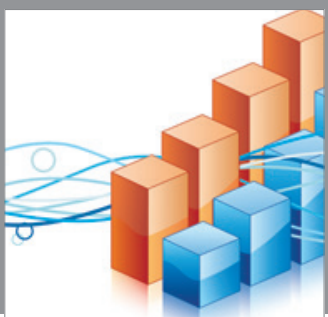

Advances in

Operations Research

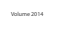

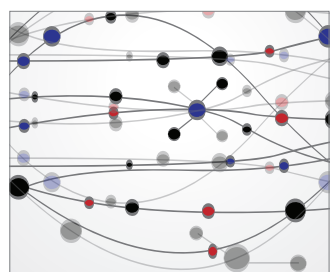

\section{The Scientific} World Journal
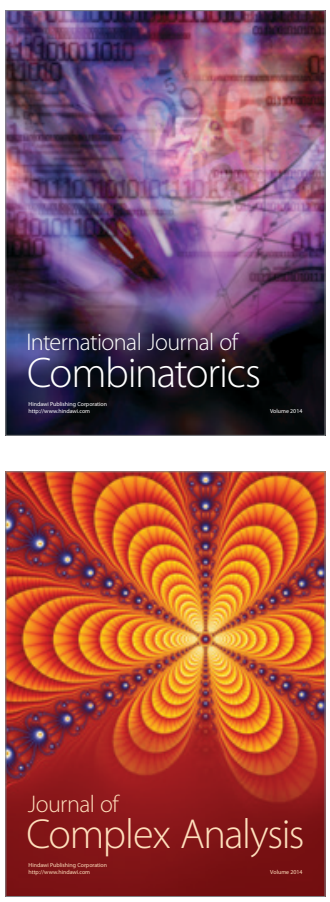

International Journal of

Mathematics and

Mathematical

Sciences
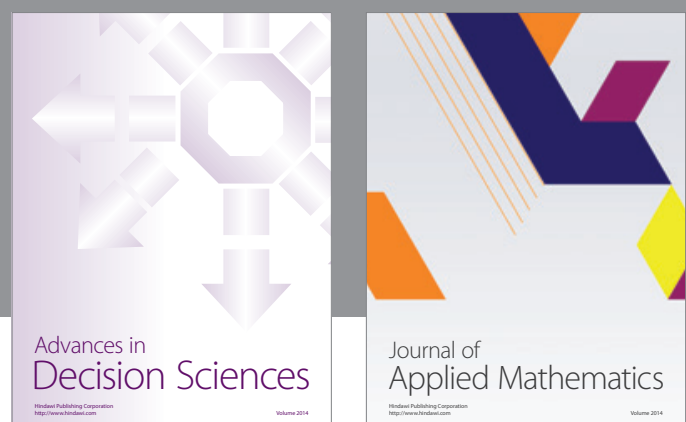

Journal of

Applied Mathematics
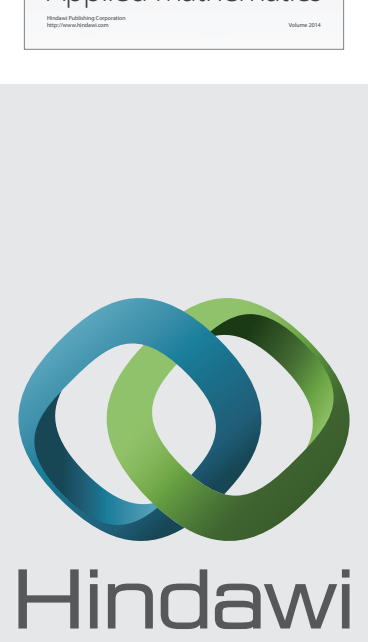

Submit your manuscripts at http://www.hindawi.com
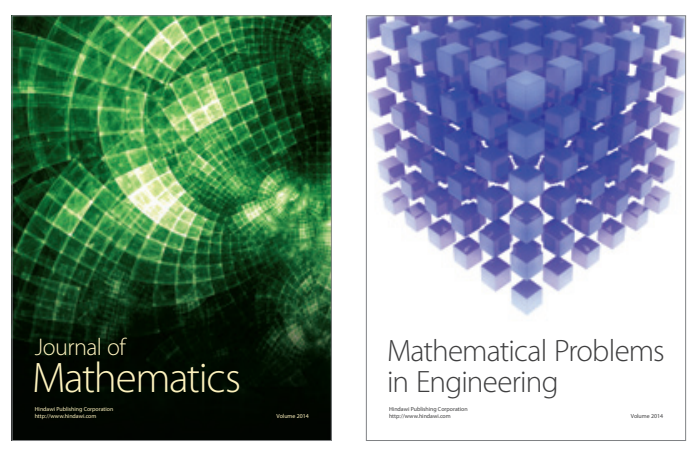

Mathematical Problems in Engineering
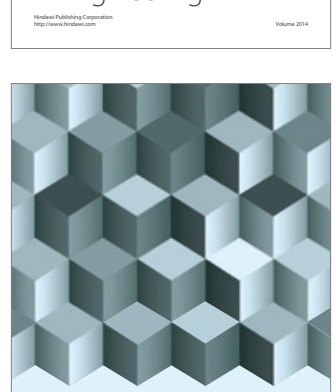

Journal of

Function Spaces
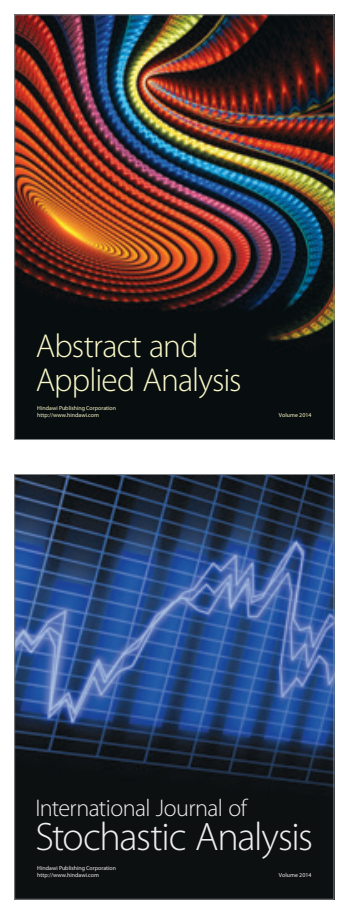

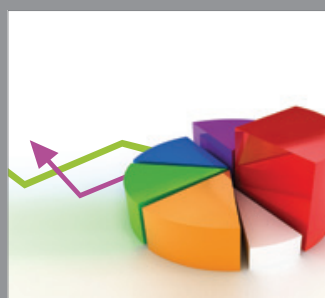

ournal of

Probability and Statistics

Promensencen
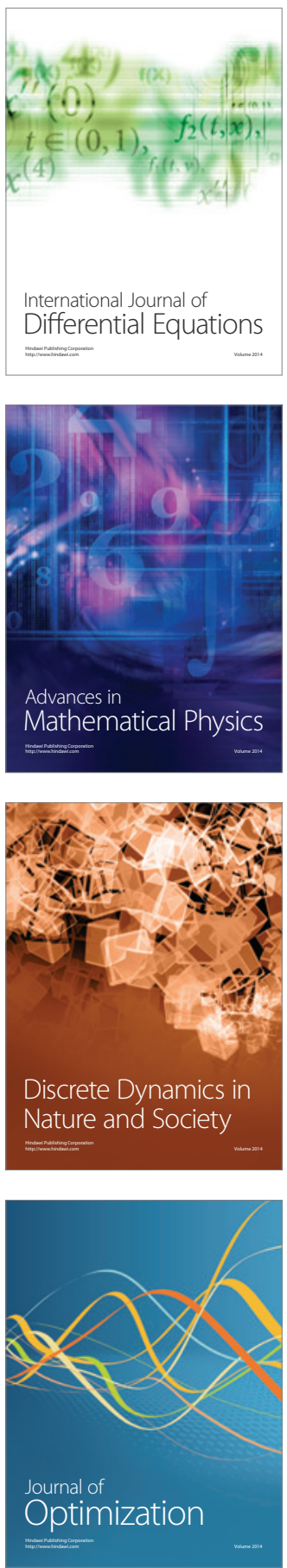